\title{
IDENTIFICACIÓN Y COMPARACIÓN DE SÍNTOMAS DE DESGASTE FÍSICO Y MENTAL EN ÁRBITROS, ENTRENADORES Y EDUCADORES FÍSICOS
}

\author{
José Luis Prendas S., Wilberth Castro C. y Gerardo Araya Vargas. \\ Escuela de Educación Física y Deportes, Universidad de Costa Rica \\ E-mail: prendas_efucr@yahoo.es,wilberth09@costarricense.cr
}

\begin{abstract}
Resumen
Prendas S., J.L.; Castro C., W. y Araya-Vargas, G.A. (2007). Identificación y comparación de síntomas de desgaste físico y mental en árbitros, entrenadores y educadores físicos. Revista de Ciencias del Ejercicio y la Salud, 5(1), 55-69. El propósito del estudio fue identificar y comparar los síntomas de desgaste físico y mental en árbitros y jueces deportivos, entrenadores deportivos y profesores (as) de educación física. Se trabajó con un total de 44 sujetos $(37,93 \pm 11,58$ años de edad), de los cuales 14 eran entrenadores, 15 profesores (as) de educación física y 15 árbitros o jueces deportivos. A todos los sujetos se les aplicó cuatro instrumentos: Perfil de Estados de Ánimo (POMS), Cuestionario de Síntomas de Sobreentrenamiento (CSSE) y Medida de Burnout (MB) y una entrevista diseñada por los investigadores. Los resultados del estudio mostraron que un $25 \%$ de los sujetos estaba en situación de riesgo ( 3 entrenadores, 5 educadores, 3 árbitros) y un 4,5\% (1 entrenador y 1 árbitro) tenían manifestaciones del burnout. Además, se encontró correlaciones significativas entre cuatro estados de ánimo (depresión, hostilidad, tensión y fatiga), tres síntomas de sobreentrenamiento (fatiga generalizada, disminución de la conducta y deseo sexual y alteraciones musculares y falta de apoyo social e irritabilidad) y el puntaje de la Escala de Burnout. Mediante análisis de $\chi^{2}$, se mostró que no había relación significativa $\left(\chi^{2}=0,169 ; p>0,05\right)$ entre el rol y el riesgo de sufrir burnout. Esto se confirmó mediante regresión logística donde se observó que la posibilidad de estar en riesgo o de sufrir el síndrome de burnout eran similares para los tres roles examinados $(1,12$ veces si se era entrenador; 1,25 veces si se era árbitro y 0,7 veces si se era profesor de educación física). Mediante análisis de varianza se encontró diferencia significativa según el nivel de riesgo en cuatro estados anímicos (depresión, hostilidad, tensión y fatiga) y cuatro síntomas de sobre entrenamiento (fatiga generalizada, falta de apoyo social e irritabilidad, deseo de asilamiento y negativismo y disminución de rendimiento). Los sujetos con riesgo o con burnout, tuvieron puntajes promedio, significativamente mayores en esas variables, en comparación con los sujetos que tenían mínimo y bajo riesgo. La evidencia muestra que en los tres grupos estudiados, existe el problema del desgaste físico y mental y estos sujetos no cuentan con asesoría adecuada para manejar esa situación. Por tanto, se sugiere atención, desde la psicología deportiva, a los diferentes grupos con que se trabajó, vigilando los estados de ánimo y síntomas de desgaste que se relacionan con la aparición del síndrome de burnout.
\end{abstract}

PALABRAS CLAVE: Burnout, estados de ánimo, sobreentrenamiento, árbitro, entrenador, educador físico.

\section{INTRODUCCION}

La práctica de deporte y ejercicio, se ha vinculado con la salud (Weinberg y Gould, 1995). Sin embargo, estas actividades no escapan a la vivencia de estados de estrés y de sus consecuencias negativas, cuando este es excesivo.
La multiplicidad de especializaciones de la práctica deportiva, la intensidad de su práctica, así como la significancia social del buen desempeño en las competiciones, entre otras cosas, conlleva a que se den determinadas respuestas psicológicas y físicas en el encargado de su puesta en práctica. 


\section{SOBREENTRENAMIENTO}

El término sobreentrenamiento, se ha aplicado principalmente al deportista o persona que entrena físicamente. Pero, su concepto va más allá y tiene gran relación con la vivencia de estrés. García, Navarro y Ruiz (1996), lo definen como una condición de fatiga y bajo rendimiento, asociada con infecciones frecuentes y depresión, la cual ocurre asociada con alta exigencia (entrenamientos y competencias fuertes, etc.). A la vez, la Asociación Médica Americana, lo define como una condición fisiológica y psicológica que se manifiesta como un estado de deterioro en la disposición atlética (Mora Segura, 2006).

Dentro de las posibles causas del síndrome de sobreentrenamiento (SSE) según García, Navarro y Ruiz (1996), con relación a nuestro objeto de estudio se encuentran: requerimientos técnicos complejos sin las pausas de recuperación necesaria, alteraciones frecuentes de los hábitos de vida por la práctica deportiva, insuficiencia de las pausas entre sesiones, solicitación demasiado elevada en la vida profesional, conflictos sociales o afectivos, excesos tóxicos (alcohol, tabaco, droga, doping), sexuales y dietéticos, alteraciones psíquicas (ansiedad, estrés, depresión, etc.), alteraciones del sueño (insomnio o factores ambientales), excesiva motivación, enfermedades (infecciones, anemias y procesos patológicos en general), estrés calórico.

\section{BURNOUT}

Freudenberger (1974) fue el primer autor que empleó el concepto de burnout para expresar las manifestaciones del síndrome en aquellos profesionales que tienen un contacto intenso con sus clientes, sobre todo, en el campo de las organizaciones.

El burnout es un síndrome que se da como producto de una exposición permanente a la vivencia de tensión, de estrés (Weinberg y Gould, 1995).

El burnout consiste, en general, en un estado de decaimiento físico, mental y emocional.
Revista de Ciencias del Ejercicio y la Salud ${ }^{\circledR}$

Vol. 5, $N^{\circ} 1,2007$

Según Almendro (2001) sus manifestaciones habituales son:

Mentales o cognitivas: sentimientos de desamparo, fracaso e impotencia. Baja autoestima. Inquietud y dificultad para la concentración. Comportamientos paranoides $\mathrm{y} / \mathrm{o}$ agresivos hacia los pacientes, compañeros y familia.

Físicas: cansancio. Dolores osteoarticulares y cefaleas. Trastornos del sueño. Alteraciones gastrointestinales, taquicardias.

De la conducta: consumo elevado de café, alcohol, fármacos y drogas ilegales. Ausentismo laboral. Bajo rendimiento personal. Conflictos interpersonales en el trabajo y el ambiente familiar.

Mora Segura (2006) cita que se ha determinado que algunas de las características de la personalidad, pueden llevar más fácilmente a la persona sometida a un exceso de estrés a desarrollar un estado de burnout. Estas características son: sensibilidad a los sentimientos y necesidades de los otros, dedicación al trabajo, idealismo, personalidad ansiosa y elevada autoexigencia.

De igual forma, Mora cita algunos de los desencadenantes del burnout como: sobrecarga de trabajo y ocupación poco estimulante, poca o nula participación en la toma de decisiones, falta de medios para realizar la tarea, excesiva burocracia (no importa el resultado, solo hacer las cosas de una determinada forma), pérdida de identificación con lo que se realiza, percepción de que no se recibe refuerzo cuando el trabajo se desarrolla eficazmente; baja expectativa de qué hacer para que el trabajo sea tenido en cuenta y valorado.

Maslach y Jackson (1981 y 1986), definieron el síndrome de burnout a partir de los síntomas que lo acompañan, destacando actitudes negativas, insensibilización, culpa, fatiga, insomnio, 
dolores de cabeza, incremento de la vulnerabilidad a las enfermedades virales, problemas digestivos, abuso de alcohol, drogas, problemas de sociabilidad, deseos de no trabajar, autoestima reducida y deterioro en los procesos cognitivos donde resalta la dificultad para la concentración. Estos autores ampliaron el campo de la investigación a otros ámbitos de la actividad humana, incluyendo el deporte.

Para Smith (1986), el burnout es un concepto complejo y consiste en una respuesta psicofisiológica exhaustiva, exhibida como resultado de esfuerzos frecuentes, a veces extremos pero en general ineficaces, que tienen por objeto satisfacer las demandas competitivas $\mathrm{y}$ del entrenamiento excesivo.

Fender (1989) definió burnout en el deporte, como una reacción al estrés de las competencias cuyos síntomas principales son: agotamiento emocional, actitud impersonal hacia los demás y disminución del rendimiento.

Weinberg y Gould (1995) consideran que, una persona pasa por una serie de etapas que le pueden llevar al burnout, estas etapas difieren dependiendo de las fuerzas y debilidades de la persona $\mathrm{y}$ de las demandas ambientales particulares en las que él o ella quizás se encuentren:

1. La despersonalización: el sujeto se muestra emocionalmente alejado, distante y desconcentrado.

2. Decrecen los sentimientos de satisfacción personal: sentimientos de poca contribución, poca satisfacción, en la actividad o deporte que practica.

3. El aislamiento: el sujeto busca las excusas para no competir o ejercitarse.

4. El agotamiento emocional y físico: el lento proceso de burnout está completo cuando, no se desea seguir desarrollando la tarea, deporte o ejercicio, ni se tienen las energías para hacerlo (alto agotamiento, ambos físico y emocional; pérdida de energía, del interés, y de la confianza; baja satisfacción personal, baja auto percepción, fracaso y depresión). Estas características, son a menudo visibles en la baja productividad del trabajo o en un nivel disminuido del desempeño.

\section{ESTADOS DE ÁNIMO}

Los estados de ánimo permiten dar una referencia sobre las causas o consecuencias del desgaste físico y mental. Según Smith (1986), los sujetos que experimentan sobreentrenamiento o agotamiento corren el riesgo de desarrollar trastornos del estado de ánimo que pueden dar como resultado una disminución de los niveles de ejecución y el abandono de la actividad. Para Smith y Crabbe (2000), los estados anímicos, son más duraderos que las emociones, que afectan a los procesos cognitivos, y resultan de cambios graduales y sostenidos del estatus neurohormonal del organismo y de la exposición prolongada a estímulos emocionales.

El instrumento más usado para su estudio en el contexto deportivo y del ejercicio es el POMS (Profile of Mood Sates). Con éste se miden los estados de:

Depresión: representa un estado de ánimo deprimido acompañado de un sentimiento de inadecuación personal; está definido por adjetivos como: desdichado, infeliz, desamparado, amargado, triste y deprimido (Fuentes, Balaguer, Meliá y García-Meritá, 1996)

Fatiga: sensación de cansancio o agotamiento que se produce después de realizar un ejercicio físico; estado de laxitud, inercia y bajo nivel de energía. Formado por los adjetivos: fatigado, agotado, cansado, exhausto y sin fuerzas (Fuentes y otros, 1996).

Hostilidad: estado de cólera y antipatía hacia los demás. Los adjetivos que lo componen son: enfadado, enojado, de mal genio, furioso, molesto e irritable (Fuentes $\mathrm{y}$ otros, 1996)

Tensión: está caracterizado por adjetivos que describen aumentos en la tensión músculo - esquelética: inquieto, intranquilo, nervioso, tenso, relajado y agitado (Fuentes y otros, 1996). 
Vigor: estado de vigor, ebullición y energía elevada. Lo describen los siguientes adjetivos: enérgico, lleno de energía, vigoroso, activo, luchador y animado (Fuentes y otros, 1996).

En síntesis, cualquier persona, sea deportista o no, que experimente estrés y no logre recuperarse adecuadamente, durante un tiempo suficientemente largo, progresivamente experimentará un desgaste de sus niveles de energía física y mental; si el desgaste se vuelve crónico, entonces aparecerán los síntomas del sobreentrenamiento y si esta condición se agrava, entonces sobrevendrá el estado de burnout y posteriormente, la posibilidad alta de abandono de la actividad, entre otras consecuencias.

\section{DESGASTE FÍSICO Y MENTAL EN ENTRENADORES}

Mora Segura (2006) cita que, por lo general, los entrenadores al igual que los deportistas se enfrentan a factores estresantes: largas sesiones de entrenamiento, elevado gasto de energía física y mental y presiones sobre el rendimiento a la hora de competir.

Para García Ucha (2000), el burnout constituye el mayor impacto de la percepción de estrés sostenido durante años de trabajo, que se expresa en el estado emocional de la persona, la cual se siente agotada, despersonalizada y reducida en su realización personal.

González, Gil y Martín (2001) citan que el entrenador, en virtud de su posición de autoridad dentro del grupo, está en posesión del control y se le percibe como alguien más válido que los otros miembros del equipo; consecuentemente, el entrenador está en condiciones para ejercer una importante influencia, de naturaleza positiva en algunos casos y negativa en otros, de allí depende entonces, que un buen o mal estado físico y mental son claves para el desarrollo de su función y de la del funcionamiento del equipo o atleta del cual está a cargo.

Kroll y Gunderscheim (1982), destacan que los entrenadores están más propensos al agotamiento pues se encuentran sometidos a la presión para ganar, interferencias e indiferencias administrativas y parentales, problemas disciplinarios, papeles múltiples a desempeñar, largos viajes e intensa implicación personal.

Quigley, Slack y Smith (1987) encontraron que el sexo de los profesoresentrenadores, edad, grado escolar, valoración del apoyo administrativo y la compensación, reconocimiento y gratificación, se relacionan con altos niveles de burnout.

Weinberg y Gould citan que las mujeres, a lo largo de los estudios realizados, son quienes se han encontrado con mayores niveles de este síndrome, de la misma forma que lo concluyen Vealey, Udry, Zimmerman y Soliday (1992E) y Kelley, Eklund y Ritter-Taylor (1999), no obstante hay controversia al respecto.

En cuanto a la edad de los entrenadores Weinberg y Gould destacan que los más jóvenes son quienes se encuentran con mayores niveles; los de mayor edad, probablemente tienen buenas técnicas de afrontamiento del estrés en su medio por su trayectoria y experiencia.

Dale y Weinberg (1989) investigaron a entrenadores de preparatoria y de colegio y encontraron que aquellos con un estilo de liderazgo interesado y orientado a las personas, tenían niveles más altos de burnout percibido que los entrenadores con mayor autoridad y con orientación de metas más definidas. Es decir que quienes trabajan de forma más personal con el atleta, tienen más tendencia al burnout porque "ellos cuidan más", lo cuál no deberá entenderse como que los entrenadores deben cuidar menos - sino, ellos deben estar enterados que este estilo requiere generalmente mucha energía y tiempo y pueden ser víctimas a largo plazo.

García Ucha (2000), en su estudio de entrenadores de equipos deportivos de alto rendimiento, encontró en estos un promedio bajo de burnout; la escala del 
inventario con mayor puntaje fue "Sobrecarga", donde el déficit de tiempo para cumplir las tareas se señaló como el factor principal. Además, los resultados del inventario se incrementaban en función de la edad y los años de experiencia en la labor. Resulta evidente que la labor de entrenador presenta un estrés sostenido capaz de generar diferentes grados potenciales del burnout.

\section{DESGASTE FÍSICO Y MENTAL EN EDUCADORES FÍSICOS}

Fejgin, Ephraty y Ben-Sira (1995) reseñan similitudes entre los predictores de burnout en entrenadores y profesores de educación física. Weinberg y Gould (1995) citan que los entrenadores en las escuelas, secundarias o en el nivel de universidad, son a menudo responsables de varios equipos y de diversas sesiones de entrenamiento, y a ello se le agrega la tensión generada por la preparación previa a competiciones.

Las investigaciones encontradas que refieran a educadores físicos no son muchas. No obstante, de las encontradas se reflejan criterios importantes de tomar en cuenta. Fejgin, Talmor y Erlich (2005) en un estudio en Grecia, sobre la integración de estudiantes con necesidades especiales en las clases de educación física (primaria), encontraron que la mayoría de los profesores contestó que las instituciones no poseen instalaciones adecuadas para los estudiantes con necesidades educativas especiales. Que se tarda demasiado tiempo para establecer un diagnóstico y darles un tratamiento específico a estos estudiantes. Hay dificultades para guiar a estos estudiantes, cuidar de su seguridad y utilizar adaptaciones curriculares para incluirlos en las clases, así como elaborar informes para sus padres. Consecuentemente se reveló que no existe fundamento sólido a la hipótesis, de que los recursos personales de los profesores y las condiciones de trabajo están directamente relacionados con el burnout. Pese a ello, se encontró también, que el número de estudiantes en clase está directamente relacionado con el burnout, la cantidad de profesores de apoyo en clase ejerce una influencia positiva en la disminución del burnout, además la mayoría de los profesores siente que la estructura y la dimensión social son irrelevantes con sus condiciones de trabajo y la mayoría de ellos mostraron estado de burnout.

Smith y Wai Leng (2003), encontraron relación entre burnout y cuatro dimensiones teóricas: psicológica, estructural-física, social y burocrática, de 74 profesores de educación física en Singapur, destacando niveles moderados de burnout, principalmente en la dimensión psicológica y social, y se encontró que no hay relación significativa entre este síndrome y los datos demográficos.

Wu (1992) en su estudio encontró que los profesores-entrenadores son más propensos a experimentar el rol de estrés en más alto nivel, por el papel de entrenador. Encontró que el excesivo tiempo y energía demandado fue el principal recurso que contribuyó con el rol de estrés, y que los que entrenan equipos deportivos son más propensos a experimentar el rol del estrés a mayores niveles.

Kelley y otros (1999) en su análisis preliminar sobre la tensión y burnout encontraron que los entrenadores de tenis de secundaria- sufrían de niveles de burnout similares a los de otros profesionales que trabajaban en educación superior.

\section{DESGASTE FÍSICO Y MENTAL EN ÁRBITROS Y JUECES DEPORTIVOS}

Las investigaciones sobre el arbitraje no son muy numerosas y las que hay se centran sobre cuestiones demasiado puntuales. Weinberg y Gould destacan que al igual que los entrenadores atléticos, los árbitros sienten roles conflictivos y tienen los niveles más altos de burnout percibido.

En el estudio de D'Ottavio y Castagna (2001), se concluye que el alto nivel de los 
árbitros de fútbol -Serie A de Italia- implica altas demandas fisiológicas sobre estos, siendo necesario entrenamientos específicos y el aumento de su aptitud física.

Los árbitros también se enfrentan a un fuerte estrés, el miedo al fracaso es el elemento que predice con mayor fiabilidad el agotamiento de los árbitros deportivos (Taylor, Daniel, Leith y Burke, 1990). Estos informan que pitar faltas y hacer amonestaciones son grandes factores de estrés relacionados con el agotamiento percibido, y que los jugadores, entrenadores y espectadores tienden a evaluarlos más de forma negativa que positiva (Mora Segura, 2006).

El arbitraje está, en general, sometido a unas altas exigencias que no permiten ningún tipo de error. Todo ello a pesar del reconocimiento público de la dificultad de la labor arbitral (Martín, 2001, citado en González y otros, 2001).

Rainey (1999) estudió las fuentes de tensión $\mathrm{y}$ de burnout en árbitros de baloncesto y el análisis de datos reveló cinco fuentes correlacionadas con los factores de la tensión: preocupaciones del funcionamiento, miedo del daño físico, carencia del reconocimiento, presión del tiempo y conflicto interpersonal. El burnout en árbitros deportivos "es consistentemente relacionado a las preocupaciones del desempeño, a la presión del tiempo, y al conflicto interpersonal, y el burnout es un predictor constante de la intención de retiro. Para Rainey, los árbitros encaran importantes compromisos y a la vez, tienen pocas compensaciones por un buen desenvolvimiento. Conllevan altas tasas de exigencia física y evidentemente, el temor del fracaso es el pronosticador más fuerte de burnout. Fuentes importantes de estrés son el hacer llamadas correctivas y que además, tanto los jugadores, entrenadores y espectadores tienden muchas veces a evaluarles negativamente.

Martín (cit. en González y otros, 2001) destaca que los errores arbitrales llevan implícito un claro perjuicio, tanto para los participantes en la práctica deportiva como para ellos mismos, pues el árbitro está permanentemente en juicio y valoración por parte de jugadores, técnicos, directivos, espectadores y medios de comunicación, además de por el Comité Técnico de Árbitros.

Kaissidis, Anshel, Tsorbatsoudis y Sideridis (1998) en su estudio, intentaron determinar las fuentes y la intensidad de la tensión aguda que se experimenta al arbitrar en el baloncesto griego. Los resultados revelaron que los árbitros relacionaron las fuentes de estrés con diferentes aspectos como "el hacer una llamada incorrecta," "carencia de la cooperación con compañeros," "presencia del supervisor," "amenazas del abuso físico," y del "abuso verbal de los entrenadores."

Respecto a investigaciones sobre el desgaste físico y mental, en Costa Rica se encontró un estudio (Pérez Rojas, 2000) sobre las fuentes de estrés y estrategias de afrontamiento en futbolistas, en el cual se utilizó el Profile of Mood States (POMS). Otro estudio costarricense, fue el de Mora Segura (2006), quien investigó los síntomas de sobreentrenamiento y burnout en futbolistas costarricenses de segunda división.

Según la reseña teórica presentada, se evidencia la problemática que viven muchas de las personas involucradas en el quehacer deportivo; por ende es el interés de la investigación, identificar y comparar los principales síntomas de desgaste físico $\mathrm{y}$ mental en las diferentes poblaciones involucradas en el área deportiva: entrenadores deportivos, árbitros o jueces deportivos y profesores de educación física.

\section{METODOLOGÍA}

\section{Sujetos}

Para el estudio se contó con la participación de 44 hombres con edades comprendidas entre los 20 y los 65 años de edad. De ellos $15(34,1 \%)$ eran árbitros o 
jueces de diferentes disciplinas deportivas; 14 $(31,8 \%)$ entrenadores de diferentes disciplinas deportivas y $15(34,1 \%)$ profesores de educación física que en el momento del estudio ejercían en algún sector del sistema de educación formal (educación primaria, educación secundaria, educación superior). La muestra tenía en promedio $37,93 \pm 11,58$ años de edad; $19,64 \pm 15,03$ horas semanales dedicadas a su rol y $10,91 \pm 8,60$ años de experiencia.

\section{Instrumentos}

A todos los sujetos se les aplicó cuatro instrumentos:

1. El Profile of Mood States (POMS): forma abreviada del perfil de estados de ánimo (Fuentes, Balaguer, Meliá y García - Meritá, 1996) que consta de 29 ítems, en donde cada uno se califica en una escala de cuatro puntos, donde 0 equivale a nada, 1 a poco, 2 moderadamente, 3 bastante y 4 muchísimo. El instrumento consta de cinco subescalas (depresión, hostilidad, tensión, vigor y fatiga) que se suman por separado de acuerdo a los ítems correspondientes.

2. Cuestionario de Síntomas de Sobreentrenamiento (CSSE) (Legros, 1993), el cual está compuesto por 28 ítems que indican si durante el último mes, los sujetos habían padecido los síntomas allí descritos.

3. Escala o Medida de Burnout (adaptación castellana de Fernández - Castro, Doval, Edo, y Santiago, 1992, 1994), que consta de 21 ítems en donde cada ítem se calificará en una escala de 7 puntos para identificar el nivel de riesgo en que puede clasificarse. El sujeto contesta cómo se siente, en una escala de siete puntos (del 1 al 7), donde 7 equivaldría a "totalmente cierto" y 1, a "totalmente falso". Al final se suman los puntajes de cada ítem para obtener la puntuación directa de la escala y luego se procede a ubicar ese puntaje, en la puntuación estandarizada.

4. Instrumento cualitativo: una entrevista cerrada diseñada por los investigadores, con la cual se recopiló datos generales de cada sujeto y diversas preguntas con las que se trató de
Revista de Ciencias del Ejercicio y la Salud ${ }^{\circledR}$

Vol. 5, $N^{\circ} 1,2007$

examinar las causas y caracterizaciones de los desgastes físico y mental en los respectivos sujetos.

\section{Procedimientos}

Cada sujeto completó las escalas y una vez terminadas, facilitó la información que requería para la entrevista. Posteriormente se procedió a realizar el análisis estadístico y un análisis de datos (cualitativo) de las entrevistas.

\section{Análisis Estadístico}

Se utilizó estadística descriptiva: frecuencias, promedios, desviaciones estándar (DS) de las diferentes variables medidas. Además se calculó frecuencias absolutas y relativas, cuando fuese necesario. Como estadística inferencial se aplicó pruebas no paramétricas $\left(\chi^{2}\right)$ y paramétricas (correlaciones de Pearson, regresión logística y análisis de varianza de una vía). Se realizó también un análisis interpretativo y crítico de la información de la entrevista.

\section{RESULTADOS}

En la Tabla 1 se muestran las frecuencias y porcentajes respectivos de los deportes en los que se desempeñaban los sujetos (árbitros y entrenadores). El fútbol fue la disciplina de la que provenían más sujetos $(58,62 \%)$.

Tabla 1. Frecuencias de los árbitros y entrenadores, según la disciplina deportiva

\begin{tabular}{ccc}
\hline $\mathrm{D}$ e p o r t e & FREC & $\%$ \\
\hline Fútbol & 17 & 58,6 \\
Voleibol & 1 & 3,44 \\
Baloncesto & 1 & 3,44 \\
Softbol & 3 & 10,3 \\
Atletismo & 2 & 6,89 \\
Boxeo & 2 & 6,89 \\
Fútbol sala & 2 & 6,89 \\
Aeróbicos & 1 & 3,44 \\
\hline Total & 29 & 100 \\
\hline
\end{tabular}


Para determinar la relación entre el rol jugado por los sujetos en estudio (entrenador, árbitro o profesional en educación física) y el nivel de riesgo de sufrir el síndrome de burnout, se corrió el análisis de $\chi^{2}$ y se encontró que no había relación significativa $\left(\chi^{2}=3,84 ; \mathrm{p}>0,05\right)$ entre esas variables. Sin embargo, como se aprecia en la tabla 2, existían celdas vacías. De hecho, un $75 \%$ de las celdas no cumplía con el requisito de no tener frecuencias inferiores a 5 . Por tanto, se procedió a integrar las categorías mínimo riesgo y bajo riesgo en una sola y lo mismo se hizo con las categorías en riesgo y con burnout y se volvió a correr el análisis. Se encontró que tampoco había relación significativa entre las variables $\left(\chi^{2}=0,169\right.$; $\mathrm{p}>0,05$ ), por lo que se confirma que el riesgo de sufrir el síndrome de burnout era similar en los tres roles examinados.
Revista de Ciencias del Ejercicio y la Salud ${ }^{\circledR}$

Vol. 5, $N^{\circ} 1,2007$

Tabla 2. Resumen de frecuencias absolutas y relativas del riesgo de sufrir el síndrome de burnout en relación con el rol practicado

\begin{tabular}{lrrlrr}
\hline & $\begin{array}{l}\text { Mínimo } \\
\text { riesgo }\end{array}$ & $\begin{array}{l}\text { Bajo } \\
\text { riesgo }\end{array}$ & $\begin{array}{l}\text { En } \\
\text { riesgo }\end{array}$ & $\begin{array}{l}\text { Con } \\
\text { burnout }\end{array}$ & Total \\
\hline Entrenador & 0 & 10 & 3 & 1 & 14 \\
Árbitro & $(0 \%)$ & $(71,4 \%)$ & $(21,4 \%)$ & $(7,1 \%)$ & $(100 \%)$ \\
& 0 & 11 & 3 & 1 & 15 \\
Educador & $(0 \%)$ & $(73,3 \%)$ & $(20 \%)$ & $(6,7 \%)$ & $(100 \%)$ \\
físico & $(6,7 \%)$ & $(60 \%)$ & $(33,3 \%)$ & $(0 \%)$ & $(100 \%)$ \\
Total & 1 & 30 & 11 & 2 & 44 \\
& $(2,3 \%)$ & $(68,2 \%)$ & $(25 \%)$ & $(4,5 \%)$ & $(100 \%)$ \\
\hline
\end{tabular}

A continuación (tabla 3) se resume la estadística descriptiva de las variables continuas del estudio, en función del rol (entrenador, árbitro o profesional en educación física) y del grado de riesgo de sufrir el síndrome que, para efectos de comparación de aquí en adelante, se dividió en dos categorías, siguiendo el procedimiento aplicado en la prueba de $\chi^{2}$ ya comentada antes.

Tabla 3. Resumen de promedios y desviaciones estándar de los años de experiencia, estados de ánimo y factores de sobre entrenamiento, medidos en entrenadores deportivos, árbitros y educadores físicos costarricenses, según el nivel de riesgo de sufrir el síndrome de burnout

\begin{tabular}{|c|c|c|c|c|c|c|}
\hline & \multicolumn{2}{|c|}{ Entrenador $(\mathrm{n}=14)$} & \multicolumn{2}{|c|}{ Árbitro $(\mathrm{n}=15)$} & \multicolumn{2}{|c|}{ Educador físico $(\mathrm{n}=15)$} \\
\hline & $\operatorname{MBR}(n=10)$ & $\begin{array}{c}\text { ERB } \\
(n=4)\end{array}$ & $\begin{array}{c}\text { MBR } \\
(n=11)\end{array}$ & $\begin{array}{c}\text { ERB } \\
(n=4)\end{array}$ & $\begin{array}{c}\text { MBR } \\
(\mathrm{n}=10)\end{array}$ & $\begin{array}{l}\text { ERB } \\
(n=5)\end{array}$ \\
\hline $\mathrm{AE}$ & $10,90 \pm 9,31$ & $15,25 \pm 12,04$ & $9,27 \pm 6,65$ & $8,75 \pm 5,91$ & $10,20 \pm 8,48$ & $14,20 \pm 12,13$ \\
\hline $\mathrm{D}$ & $1,50 \pm 1,71$ & $4,00 \pm 5,40$ & $1,36 \pm 1,96$ & $6,25 \pm 7,13$ & $1,50 \pm 2,72$ & $7,00 \pm 2,74$ \\
\hline $\mathrm{H}$ & $2,20 \pm 3,39$ & $3,25 \pm 3,40$ & $4,27 \pm 5,16$ & $4,50 \pm 5,26$ & $3,10 \pm 3,41$ & $10,40 \pm 4,22$ \\
\hline $\mathrm{T}$ & $5,80 \pm 3,05$ & $8,25 \pm 2,87$ & $6,54 \pm 3,27$ & $8,50 \pm 4,12$ & $5,60 \pm 3,75$ & $12,80 \pm 2,95$ \\
\hline V & $16,60 \pm 3,40$ & $14,50 \pm 5,07$ & $14,64 \pm 5,94$ & $15,50 \pm 6,45$ & $15,70 \pm 6,34$ & $14,00 \pm 3,74$ \\
\hline $\mathrm{F}$ & $3,10 \pm 3,03$ & $5,25 \pm 3,59$ & $4,54 \pm 3,47$ & $7,25 \pm 6,18$ & $3,30 \pm 3,23$ & $10,00 \pm 3,81$ \\
\hline F1 & $2,40 \pm 1,95$ & $5,75 \pm 4,03$ & $3,18 \pm 2,04$ & $6,50 \pm 5,69$ & $3,00 \pm 2,87$ & $7,60 \pm 3,05$ \\
\hline F2 & $1,60 \pm 1,78$ & $3,00 \pm 1,82$ & $2,36 \pm 2,01$ & $3,00 \pm 2,94$ & $1,60 \pm 1,95$ & $3,40 \pm 3,58$ \\
\hline F3 & $1,70 \pm 1,64$ & $4,25 \pm 2,36$ & $2,64 \pm 1,63$ & $3,25 \pm 3,20$ & $2,80 \pm 2,53$ & $5,20 \pm 3,11$ \\
\hline F4 & $1,50 \pm 1,58$ & $3,00 \pm 1,15$ & $1,36 \pm 0,81$ & $2,00 \pm 2,16$ & $1,80 \pm 2,04$ & $4,00 \pm 2,34$ \\
\hline F5 & $1,60 \pm 1,17$ & $2,25 \pm 1,71$ & $2,18 \pm 1,54$ & $3,25 \pm 2,06$ & $2,60 \pm 1,26$ & $4,40 \pm 2,51$ \\
\hline
\end{tabular}

MBR: mínimo y bajo riesgo de burnout; ERB: en riesgo o con el síndrome de burnout; AE: años de experiencia en el rol; D: depresión; H: hostilidad;

T: tensión; V: vigor; F: fatiga; F1: fatiga generalizada; F2: disminución de conducta y deseo sexual y alteraciones musculares; F3: falta de apoyo social e irritabilidad; F4: deseo de aislamiento; F5: disminución del rendimiento. 
Tabla 4. Resumen de correlaciones de Pearson de la relación entre años de experiencia, estados de ánimo, factores de sobre entrenamiento y burnout

\begin{tabular}{|c|c|c|c|c|c|c|c|c|c|c|c|}
\hline & $\mathrm{D}$ & $\mathrm{H}$ & $\mathrm{T}$ & $\mathrm{V}$ & $\mathrm{F}$ & F1 & $\mathrm{F} 2$ & F3 & F4 & F5 & $\mathrm{B}$ \\
\hline$\overline{\mathrm{AE}}$ & 0,122 & 0,043 & 0,116 & $-0,131$ & 0,072 & 0,241 & $0,384 *$ & 0,255 & $0,341^{*}$ & 0,153 & 0,133 \\
\hline D & & $0,711 * *$ & $0,744 * *$ & $-0,346^{*}$ & $0,673^{* *}$ & $0,683 * *$ & $0,512 * *$ & $0,508 * *$ & $0,605^{* *}$ & $0,541^{* *}$ & $0,618^{* *}$ \\
\hline $\mathrm{H}$ & & & $0,722 * *$ & $-0,343^{*}$ & $0,715^{* *}$ & $0,512 * *$ & $0,357 *$ & $0,391 * *$ & $0,409 * *$ & $0,357^{*}$ & $0,319 *$ \\
\hline $\mathrm{T}$ & & & & $-0,313^{*}$ & $0,686 * *$ & $0,670^{* *}$ & $0,512 * *$ & $0,588 * *$ & $0,523^{* *}$ & $0,582 * *$ & $0,461^{* *}$ \\
\hline V & & & & & $-0,303^{*}$ & $-0,230$ & $-0,268$ & $-0,214$ & $-0,409 * *$ & $-0,346 *$ & $-0,094$ \\
\hline $\mathrm{F}$ & & & & & & $0,666^{* *}$ & $0,428 * *$ & $0,491 * *$ & $0,330 *$ & $0,489 * *$ & $0,499 * *$ \\
\hline F1 & & & & & & & $0,713^{* *}$ & $0,767 * *$ & $0,662 * *$ & $0,605^{* *}$ & $0,504^{* *}$ \\
\hline F2 & & & & & & & & $0,665 * *$ & $0,569 * *$ & $0,542 * *$ & $0,329 *$ \\
\hline F3 & & & & & & & & & $0,665^{* *}$ & $0,585^{* *}$ & $0,394^{* *}$ \\
\hline F4 & & & & & & & & & & $0,468 * *$ & 0,260 \\
\hline F5 & & & & & & & & & & & 0,260 \\
\hline
\end{tabular}

${ }^{*} \mathrm{p}<0,05{ }^{* *} \mathrm{p}<0.01$

AE: años de experiencia en el rol; D: depresión; H: hostilidad; T: tensión; V: vigor; F: fatiga; F1: fatiga generalizada; F2:

disminución de conducta y deseo sexual y alteraciones musculares; F3: falta de apoyo social e irritabilidad; F4: deseo de

aislamiento; F5: disminución del rendimiento; B: burnout (puntos)

Como se aprecia en la tabla anterior (tabla 4), los años de experiencia en el ejercicio del rol, se relacionaron significativamente solo con dos factores de sobreentrenamiento (disminución de conducta y deseo sexual y alteraciones musculares, $y$ el deseo de aislamiento). Por otro lado, cabe destacar que las variables que sí tuvieron correlación significativa con los puntajes de burnout fueron la depresión, la hostilidad, la tensión, la fatiga, y tres síntomas de sobre entrenamiento (fatiga generalizada, disminución de conducta y deseo sexual y alteraciones musculares y la falta de apoyo social e irritabilidad). Es decir que, a mayor grado de esas variables, mayor puntaje de burnout se presentaba en los sujetos estudiados.

Además, se corrió un análisis de regresión logística, empleando como variable dependiente binaria al riesgo de sufrir burnout $(0=$ mínimo y bajo riesgo; $1=$ en riesgo o con burnout). Este análisis permite establecer las posibilidades de que se presente el fenómeno de interés (en este caso el riesgo o la presencia del síndrome de burnout), al estar presente cada una de las variables predictoras (en este caso se incluyeron los años de experiencia, los estados anímicos medidos y los cinco síntomas de sobre entrenamiento, además se incluyó al rol como variable categórica corriéndose un modelo donde se tomaba como referencia al rol de entrenador y otro modelo donde se tomó como referencia al rol de profesor de educación física).

Los resultados mostraron que el modelo con esas variables predictoras, tuvo un buen ajuste $\left(\chi^{2}=14,728 ; p>0,05\right)$, con una precisión del $88,64 \%(93,55 \%$ de los sujetos se clasificó correctamente en el nivel de mínimo y bajo riesgo, mientras que un $76,92 \%$ de los sujetos se clasificó correctamente en el nivel de riesgo o de burnout), no obstante, las posibilidades de que el fenómeno (riesgo o presencia del síndrome de burnout) se diera son parecidas para cada una de las variables incluidas en el modelo (las posibilidades fueron en todas las variables menores a 2 veces y no fueron significativamente diferentes de cero). Así, por ejemplo, por cada año de experiencia en el ejercicio del rol (no se diferenció entre estos) el riesgo de sufrir burnout aumentaba en 1,05 veces; por cada punto de depresión, el riesgo aumentaba en 1,53 veces; por cada punto en fatiga generalizada, el riesgo aumentaba en 1,46 veces (las demás posibilidades para las otras variables, eran muy pequeñas en comparación con estas que, de por sí, ya son pequeñas).

Con respecto a los roles, se observó que la posibilidad de estar en riesgo o de sufrir el 
síndrome de burnout eran similares $(1,12$ veces si se era entrenador; 1,25 veces si se era árbitro y 0,7 veces si se era profesor de educación física). Los análisis de varianza aplicados, confirman varios de estos hallazgos.

Según la tabla 5, se encontraron diferencias significativas solo relacionadas con el nivel de riesgo de desarrollar el síndrome de burnout y no así con el tipo de rol jugado por los sujetos. Las variables en las que se encontró diferencia significativa según el nivel de riesgo fueron cuatro estados anímicos (depresión, hostilidad, tensión y fatiga) y cuatro síntomas de sobre entrenamiento (fatiga generalizada, falta de apoyo social e irritabilidad, deseo de asilamiento y negativismo y disminución de rendimiento). En estos casos, los sujetos con riesgo o con la presencia del síndrome de burnout, tuvieron puntajes promedio, significativamente mayores en esas variables, en comparación con los sujetos que tenían mínimo y bajo riesgo.

Tabla 5. Resumen de análisis de varianza aplicados a los años de experiencia, estados de ánimo y factores de sobre entrenamiento, según el rol y el nivel de riesgo de burnout

\begin{tabular}{crrr}
\hline $\begin{array}{c}\text { Variable } \\
\text { dependiente }\end{array}$ & $\begin{array}{c}\text { F de } \\
\text { Rol }\end{array}$ & \multicolumn{1}{c}{$\begin{array}{c}\text { F de } \\
\text { Riesgo }\end{array}$} & $\begin{array}{c}\text { F de } \\
\text { Rol X } \\
\text { Riesgo }\end{array}$ \\
\hline AE & 0,681 & 0,783 & 0,278 \\
D & 0,677 & $\mathbf{1 5 , 8 2 9 * *}$ & 0,710 \\
H & 2,913 & $\mathbf{4 , 2 5 1 *}^{*}$ & 2,720 \\
T & 1,455 & $\mathbf{1 2 , 0 8 6 * *}^{*}$ & 2,369 \\
V & 0,055 & 0,307 & 0,270 \\
F & 1,452 & $\mathbf{1 0 , 0 5 4 *}^{*}$ & 1,455 \\
F1 & 0,532 & $\mathbf{1 4 , 6 2 3 * *}$ & 0,194 \\
F2 & 0,084 & 2,962 & 0,215 \\
F3 & 0,896 & $\mathbf{6 , 1 0 2 *}$ & 0,673 \\
F4 & 1,659 & $\mathbf{6 , 7 6 2 *}$ & 0,680 \\
F5 & 2,991 & $\mathbf{4 , 8 8 *} *$ & 0,414
\end{tabular}

$* \mathrm{p}<0,05 * * \mathrm{p}<0.01$

AE: años de experiencia en el rol; D: depresión; H: hostilidad; T: tensión; V: vigor; F: fatiga; F1: fatiga generalizada; F2: disminución de conducta y deseo sexual y alteraciones musculares; F3: falta de apoyo social e irritabilidad; F4: deseo de aislamiento; F5: disminución del rendimiento
Revista de Ciencias del Ejercicio y la Salud ${ }^{\circledR}$

Vol. 5, $N^{\circ} 1,2007$

\section{ANÁLISIS CUALITATIVO DE LA ENTREVISTA APLICADA}

De los entrenadores que obtuvieron un puntaje catalogador de Situación de Riesgo, algunas de las más importantes caracterizaciones son las siguientes: un sujeto destacó que su labor es más complicada con los jóvenes, "pues su hiperactividad $e$ indisciplina, requiere de más paciencia, mayor gasto emocional y a la vez exige riesgos, principalmente con las mujeres, las lesiones, entre otras" (entrenador de atletismo). Otro sujeto reseñó que es más difícil trabajar con adolescentes y adultos (entrenador de fútbol sala); otro reseña específicamente la complicación con la población de 16 a 18 años (entrenador de fútbol).

Esos tres sujetos respondieron afirmativamente la pregunta: ¿ha influido el tipo de población con la que ha trabajado, en su estado anímico y en su vida personal? Los mismos, reportaron sentirse en ocasiones con estrés, aunque todos respondieron que dichas situaciones no son muy frecuentes. Los momentos que ellos describen como propiciadores para el estrés son: "en ocasiones de actividad competitiva, o por lesiones de algún competidor, así como por alguna baja en el rendimiento", "cuando no se presentan a juegos o entrenamientos", "en el momento de tomar medidas disciplinarias".

Los tres sujetos en Situación de Riesgo respondieron sentirse satisfechos de su función. Identificaron como principales inconvenientes o problemas en el desempeño de su función: la falta de apoyo -institucionalprincipalmente económico, incomprensión por parte de los atletas, sacrificios que parecen "nulos", pues no se da una verdadera valoración, la falta de aporte económico para gastos pertinentes al desarrollo de su función, poca comprensión de padres de familia, instalaciones en mal estado.

Los tres contestaron negativamente a que esos problemas formasen parte de su vida cotidiana o los afectara personalmente. De igual manera, contestaron directamente que no 
se encontraban físicamente ni psicológicamente agotados de su rol.

Respecto al entrenador clasificado en Estado de Burnout, los datos que pueden sugerir notable diferenciación son: labor de lunes a viernes durante 8 horas como entrenador de fútbol, lo hace "porque es lo que sé hacer", tiene 19 años de labor en dicho campo. Reportó que el ejercicio de su rol le ha generado bastante estrés y destaca que no son frecuentes las situaciones, pero que sí influyen en su vida personal. Se siente satisfecho de su papel. Respondió afirmativamente a encontrarse agotado tanto psicológicamente como físicamente.

En los educadores físicos, quienes obtuvieron la clasificación de Situación de Riesgo, reportaron dentro de las poblaciones más complicadas para desempeñar su función a: niños y jóvenes; un educador destacó que es difícil "al creer ellos que todo lo saben. También si se habla de pérdida de valores; los jóvenes son más permeables a esta situación." Otro educador destacó que su trabajo es más complicado "cuando parecen desinteresados, indiferentes, sin metas, sin ganas de luchar por lo que vale la pena en la vida."

Dicha argumentación se puede ver relacionada con la posición de Weinberg y Gould (1995), los cuales hacen referencia a que mientras más involucrado se está con los atletas, en este caso, estudiantes, habrá mayor tendencia hacia el síndrome de burnout. Es decir, la preocupación evidenciada es un pronosticador importante de agotamiento, en este caso de una forma mayor pues el sujeto cita el desinterés del estudiantado.

De los cuatro sujetos en Situación de Riesgo, uno respondió que sí ha pensado en abandonar su profesión, otro respondió que en algunas ocasiones lo ha pensado y dos de ellos respondieron que nunca. A la vez, uno de estos citó que la educación física "no nos da dinero (solo lo suficiente), pero sí, muchas alegrías y felicidad."

Con respecto al tipo de población y el estado anímico, un educador físico citó que se dio cuenta que desempeñaba mejor su labor con los niños en comparación con los jóvenes, "soy un poco ligero de carácter y perdía la ecuanimidad."

Otro educador afirmó que sí influía en ocasiones la población en su estado anímico y hasta citó sentimientos de frustración a causa de ello. Del mismo modo otro educador, reseñó que algunas relaciones con otras personas "a veces es una copia de la influencia de los jóvenes."

Bajo la misma perspectiva, pero adoptando una posición importante de tomar en cuenta, un educador reseñó que los estudiantes "me han hecho cambiar... ahora trato de dar lo mejor de mí, sin procurar molestarme por el desinterés que a veces muestran algunos estudiantes."

Tres de los educadores en Situación de Riesgo reportaron sentirse satisfechos de su labor y tan solo un sujeto respondió que se sentía satisfecho "a medias" y hacía referencia a que: sí, por sus intenciones propuestas, "pero no, con haber sido explícita o ellos (los estudiantes) no han captado el mensaje."

Dentro de los principales inconvenientes o problemas en el desempeño de sus funciones, los educadores físicos citaron: falta de tiempo (involucramiento de otras labores), salud (personal), falta de estímulos externos, reconocimiento a su labor (no precisamente dinero), falta de infraestructura adecuada para las clases.

Referente a los árbitros $\boldsymbol{y}$ jueces deportivos un sujeto se clasificó en Estado de Burnout; este mismo era árbitro de fútbol, pero lo ejercía como una disciplina complementaria a su labor; consideraba que no se habían cumplido muchos de sus objetivos que vislumbraba para el futuro. Los causantes de estrés estaban acorde a la ocasión y al hecho de "conocer a los equipos" que debe dirigir en los partidos. Cita que el fracaso influye parcialmente, y a veces de manera degradante en su vida personal. Relata que la población con que le corresponde trabajar, sí influye en su estado anímico. El estrés es evidenciado no tan frecuentemente; influye el hecho de "poder desempeñar un trabajo que no necesita de ser bachiller", con lo cual evidencia cierta baja en su autoestima con respecto a sus logros académicos. No se siente en la actualidad, satisfecho de su papel, no queriendo decir que se sienta mal por lo logrado, ello lo aclara al citar: "creo ser capaz de algo más." 
No destacó algún inconveniente externo en el desempeño de su labor, sino que se refirió al hecho de ser $\mathrm{o}$ no aceptado por los dirigentes y espectadores en el arbitraje de partidos. Ello lo expresó citando que dicho inconveniente sí se hace parte de su vida cotidiana.

Respondió sentirse "a veces" psicológicamente agotado, mientras que en el aspecto físico respondió que sí se siente agotado pero "no mucho".

De los tres árbitros que se clasificaron en Situación de Riesgo se puede realizar una descripción detallada de cada uno. Dos, eran árbitros de fútbol y uno de fútsal. El primero tenía 21 años, soltero y estudiante universitario; su motivo de ejercimiento era "la pasión por el fútbol." El segundo sujeto (fútsal) tenía 47 años, estaba divorciado y cursó hasta quinto año de secundaria, se desempeña también como operario industrial y su motivo de ejercimiento era "el gusto que le tengo al deporte." El tercer sujeto, tenía 39 años, estaba casado, tenía un grado académico universitario y se desempeñaba también como contador público; relata que se inició "como diversión, como hoobie" y posteriormente se involucró más de lleno. Indicó que ha seguido un debido proceso (ANAFA, Juegos Nacionales, $3^{\circ}$ División, $2^{\circ}$ División y $1^{\circ}$ División), el cual a la vez ha sido el logro de sus diferentes objetivos. No obstante reseña, que le hizo falta ser designado como árbitro de un mundial de fútbol y ello a causa de su avanzada edad.

Dentro de las poblaciones que ellos consideran más complicadas para desempeñar su labor, se encuentran las siguientes: canchas abiertas, tercera división y dependiente según la particular ocasión. Dos de ellos relatan cómo el hecho de arbitrar en diferentes poblaciones, tiene un mayor riesgo físico, esencialmente de ser agredido. Uno de ellos destaca, que pese a la degradación de la imagen pública que se da en primera división, es preferible al riesgo de violencia física de las demás categorías. Este mismo sujeto fue el único en responder que espera un pronto abandono de su profesión como árbitro. Dos de los árbitros consideran que el tipo de población sí influye en su estado anímico. Dos reportaron estrés pero no frecuentemente e hicieron alusión al momento particular después de los partidos o luego de algún conflicto. Los tres sujetos respondieron afirmativamente al cuestionamiento, de sentirse satisfechos en la actualidad de su función.

Dentro de los inconvenientes que los tres sujetos destacan en el desempeño de su función, se encuentra lo siguiente: presión de aficionados, presión de jugadores, presión propia, poca afectividad y poca confianza en la relación entre árbitros, falta de psicólogos.

Dos de los sujetos reportaron que estos problemas afectan en su vida cotidiana, mientras que el otro detalló que no afecta, pues se vuelve algo normal, "es parte de la rutina."

A las preguntas sobre el sentirse agotado, dos sujetos respondieron que no tanto física como psicológicamente, mientras que el otro sujeto respondió sentirse agotado en el plano emocional y "bastante" en el aspecto físico.

\section{DISCUSIÓN}

Los resultados mostraron que un $4,5 \%$ de la población total se encontraba en Estado de Burnout. Esto corresponde a dos sujetos, un árbitro y un entrenador deportivo. De igual forma, en la clasificación Situación de Riesgo de Burnout, los árbitros se encuentran ahí en un $20 \%$, mientras que los entrenadores evidencian un dato similar con 21,43\%. Weinberg y Gould (1995) reseñan que existe cierta relación en los niveles más altos de burnout entre entrenadores y árbitros, ello por los roles conflictivos que se desencadenan en la escena deportiva.

Previo al análisis siguiente, es importante citar que la gran mayoría de los sujetos conllevan sus labores como árbitros, entrenadores y educadores físicos (en menor grado), de la mano de otra profesión. La tendencia de tener una vida profesional exigente, es un aspecto que para Mora Segura (2006) es una característica en la personalidad de sujetos propensos al síndrome de desgaste físico y mental.

No existieron diferencias en los puntajes de burnout de las tres profesiones estudiadas ni en su posibilidad de sufrir ese estado (análisis de regresión logística), con lo cual se puede 
hacer referencia a Fejgin y otros (1995) que destacan similitudes entre los predictores de burnout en entrenadores y profesores de educación física.

Los resultados obtenidos en la población de entrenadores muestran cierta concordancia con García Ucha (2000), al mostrar como promedio la clasificación de bajo potencial de burnout, mientras que en el presente estudio un total de $71,43 \%$ mostró encontrarse en dicha clasificación. El estudio de García Ucha reveló que los resultados se incrementan en función de la edad y los años de experiencia en la labor, no obstante, los resultados mostraron que los años de experiencia no se relacionaron significativamente con el puntaje de burnout (solo se relacionaron con dos factores de sobreentrenamiento), en lo cual se difiere de García Ucha. En ese sentido, también esto es contrario a lo planteado por Weinberg y Gould (1995). No necesariamente, el tener mayor experiencia va a determinar que se hallan adquirido más herramientas para enfrentar el estrés; la forma en la que cada quien vive su experiencia (su calidad) es lo que podría hacer la diferencia.

Se encontró correlaciones significativas entre cuatro estados de ánimo (depresión, hostilidad, tensión y fatiga), tres síntomas de sobreentrenamiento (fatiga generalizada, disminución de la conducta y deseo sexual y alteraciones musculares y falta de apoyo social e irritabilidad) y el puntaje de la Escala de Burnout. Además, mediante análisis de varianza se encontró diferencia significativa según el nivel de riesgo en cuatro estados anímicos (depresión, hostilidad, tensión $\mathrm{y}$ fatiga) y cuatro síntomas de sobre entrenamiento (fatiga generalizada, falta de apoyo social e irritabilidad, deseo de asilamiento y negativismo y disminución de rendimiento). Los sujetos con riesgo o con burnout, tuvieron puntajes promedio, significativamente mayores en esas variables, en comparación con los sujetos que tenían mínimo y bajo riesgo. Estos resultados respaldan los hallazgos de Mora Segura (2006) en futbolistas.

Según Mora Segura, se destacan como desencadenantes de burnout, la sobrecarga de trabajo y ocupación poco estimulante, reflejada en las alteraciones anímicas. De igual forma en los árbitros, la tensión es una característica notable, esencialmente por su desenvolvimiento en la competición y en las repercusiones externas por su desempeño. Las caracterizaciones dadas en el instrumento cualitativo por los árbitros permiten observar cierta concordancia con las fuentes de tensión y de estrés citadas por Rainey (1999)

Respecto a las alteraciones musculares y la baja en la conducta sexual, puede existir una evidencia clara en las demandas físicas y psíquicas que se requieren en el arbitraje (D'Ottavio y Castagna, 2001) y el elevado gasto de energía en entrenadores por las largas sesiones de entrenamiento y competición (Mora Segura, 2006), también puede influir las diferentes implicaciones del arbitraje como "fuerte estrés y miedo al fracaso" (Taylor, y otros, 1990), así como el reconocimiento y devaluación pública (Martín, cit. en González y otros, 2001).

Se debe tener en consideración los niveles de autoexigencia, dedicación al trabajo, sobrecarga de trabajo, condiciones laborales, excesiva burocracia, pues todos ellos fueron evidenciados en el análisis del instrumento cualitativo, en las tres distintas poblaciones. A la vez, uno de los datos sugerentes evidenciados en el instrumento cualitativo (por una gran mayoría de los sujetos, independientemente de la profesión) y respaldado teóricamente (Fejgin y otros, 2005; Mora Segura, 2006) como un desencadenante de burnout es la falta de apoyo - aporte económico, de instalaciones y materiales.

La población encargada de dirigir deportes o programas de ejercicio físico, ejerce roles de liderazgo en contextos que le exigen superarse constantemente y dar un buen desempeño tanto físico como mental. Se ha mostrado que, las personas sometidas a estrés y que por ende tienden más al desarrollo de burnout, muestran mayores niveles de auto exigencia, lo cual es planteado por Mora Segura (2006) en su estudio.

En conclusión, pese a que los resultados no son alarmantes, se pudo demostrar la existencia de casos de entrenadores, árbitros y profesionales de educación física, que 
presentan síntomas de desgaste físico y mental que les pone en alto riesgo o en el estado manifiesto de burnout. Es necesario, extender este campo de estudio que se ha enfocado principalmente en atletas y poner mayor atención a las necesidades de las personas que dirigen los procesos de preparación deportiva, de entrenamiento físico y los espectáculos deportivos. La evidencia muestra que en los tres grupos estudiados, existe el problema del desgaste físico y mental y estos sujetos no cuentan con asesoría adecuada para manejar esa situación. Por tanto, se sugiere atención, desde la psicología deportiva, a los diferentes grupos con que se trabajó, vigilando los estados de ánimo y síntomas de desgaste que se relacionan con la aparición del síndrome de burnout.

\section{REFERENCIAS}

Almendro, C. (2001). Plataforma 10_Minutos. Recopilado por Mora Segura, M., el 7 de septiembre 2004. En http://www webmaster@diezminutos

Dale, J. y Weinberg, R. (1989). Burnout in sport: A review and critique. Journal of Applied Sport Psychology, 2: 67 - 83.

D'Ottavio, S. y Castagna, C. (2001) Physiological load imposed on elite soccer referees during actual match play. Journal of Sports Medicine and Physical Fitness, 41(1): 27-32.

Fejgin, N., Ephraty, N. y Ben-Sira, D. (1995). Work environment and burnout of physician education teachers. Journal of Teaching in Physical Education. 15: 64-78. .,Talmor, R. y Erlich, I. (2005). Inclusion and burnout in physical education. European Physical Education Review, 11(1): $29-50$

Fender, L. (1989). Athlete burnout: Potential for research and interventions strategies. The Sport Psychologist, 3(1):6371.

Fernández - Castro, J., Doval, E., Edo, S. y Santiago, M. (1992). L'estrés docent dels mestres de Catalunya. Barcelona. Informe del departament d'Ensenyament.

Fernández - Castro, J., Doval, E., Edo, S. y Santiago, M. (1994). Efectos del estrés docente sobre los hábitos de salud. Ansiedad y Estrés. pp.: 127-133.

Freudenberger, H. J. (1974). Staff burn-out. The Journal of Social Issues. 30 (1):159-166.

Fuentes, I.; Balaguer, I.; Meliá, J. L. y García-Meritá, M. L. (1996). Forma abreviada del perfil de estados de ánimo (POMS). Actas del V congreso Nacional de Psicología de la Actividad Física y el Deporte, Valencia, España, 1995, p. 19-26.

García, Navarro y Ruiz. (1996).Bases teóricas del entrenamiento deportivo. Gymnos Editorial. Madrid, España.

García Ucha, F. (2000). Entrenadores y Burnout. Revista Digital Efdeportes Buenos Aires - Año 5 - $\mathrm{N}^{\mathrm{o}} 28$.
Revista de Ciencias del Ejercicio y la Salud ${ }^{\circledR}$

Vol. 5, $N^{\circ} 1,2007$

Obtenido 13 de marzo del 2006 en: http://www.efdeportes.com.

González, J., Gil, C. y Martín, G. (2001). Manual de prácticas de psicología deportiva. 1 ed. Madrid, España: Editorial Biblioteca Nueva, S. L.

Kaissidis, R., Anshel, M., Tsorbatsoudis, C. y Sideridis, G. (1998). Sources and intensity of acute stress in Greek basketball referees: a preliminary study. Exercise \& Society Journal of Sport Science, 19: 54-68

Kelley, B., Eklund, R.C. y Ritter-Taylor, M. (1999). Stress and Burnout Among Collegiate Tennis Coaches. Journal of Sport \& Exercise Psychology, 21(2): : 113-130

Kroll, W. y Gunderscheim, J. (1982). Stress factors in coaching. Coaching Science Update, pp.: 47-49.

Legros, P. (1993). Le surentrainement: diagnostic des manifestations psychocomportementales précoces. Science and Sports, 8: 71-74.

Maslach, C., y Jackson, S.E. (1981). The Maslach Burnout Inventory. Research edition.Consulting Palo Alto, C.A.: Psychologists Press.

Maslach, C., \& Jackson, S.E. (1986).The Maslach Burnout Inventory. 2 ed. Palo Alto, C.A.: Consulting Psychologists Press.

Mora Segura, M. (2006). Identificación de síntomas de sobreentrenamiento y burnout en futbolistas varones costarricenses de segunda división en función de los estados anímicos, apoyo percibido de figuras significativas, edad de inicio, experiencia competitiva y desempeño colectivo. Tesis (sin publicar) Licenciatura en Ciencias del Deporte. Universidad Nacional. Heredia, Costa Rica.

Pérez Rojas, A. (2000). Fuentes de estrés y estrategias de afrontamiento en un grupo de futbolistas de primera división. Tesis de licenciatura en psicología. San José, Costa Rica. Universidad de Costa Rica.

Quigley, T.A.; Slack, T. y Smith, G.J. (1987). Burnout in secondary-school teacher coaches. Alberta Journal of Educational Research (Edmonton), 33(4): 260274

Rainey, D. (1999). Sources of stress, burnout, and intention to terminate among basketball referees. Journal of Sport Behavior, 22(4): 578-590.

Smith, R.E. (1986). Toward a cognitive-affective model of athletic burnout. Journal of Sport Psychology, 8(1): 36-50.

Smith, D. y Wai Leng, G. (2003). Prevalence and Sources of Burnout in Singapore Secondary School Physical Education Teachers. Journal of Teaching in Physical Education, 22(2).

Smith, J. C. y Crabbe, J. B. (2000). Emotion and exercise. International Journal of Sport Psychology, 31: 156 -174 .

Taylor, A. H., Daniel, J. V., Leith, L. y Burke, R. J. (1990). Perceived stress, psychological burnout and paths to turnover intentions among sport offials. Journal of Applied Sport Psychology, 2: 84 - 97. 
Vealey R., Udry E., Zimmerman V. y Soliday J. (1992). Intrapersonal and situational predictors of coaching burnout. Journal of Sport and Exercise Psychology, 14(1): 40-58.

Weinberg, R. y Gould, D. (1995). Foundations of sport and exercise psychology. 1 ed. Champaign, IL: Human Kinetics.

Wu, Y. (1992). Role stress and secondary school teacher/coaches in the state of Washington. Publisher: Eugene, Ore. : Microform Publications, College of Human Development and Performance, University of Oregon.

Fuente: http://kinpubs.uoregon.edu/MFPBull7pp151198.pdf. Obtenido: mayo de 2006. 